\title{
Prediction of losses in belt-type continuously variable transmission due to sliding between belt and disc
}

\author{
G Poll, T Kruse, and C Meyer \\ Department of Machine Elements, Institute for Machine Elements, Engineering Design and Tribology, \\ University of Hannover, Hannover, Germany
}

The manuscript was received on 1 September 2005 and was accepted after revision for publication on 10 February 2006.

DOI: 10.1243/13506501JET141

\begin{abstract}
The efficiency of belt-type continuously variable transmission (CVT) - apart from the power consumption of auxiliary systems such as hydraulics - predominantly depends on the energy dissipated during sliding at the belt-disc contacts. These sliding motions are a consequence of elastic deformations and misalignments because of clearances, resulting in a socalled spiral path of the belt elements on the discs and hence tangential and radial sliding motions.

The performance of such systems can be predicted through an iterative computation by numerically solving a set of differential equations for the forces and motions coupled with a finite-element computation of the deformations.

A comparison with elaborate measurements of deflections, belt motions, and losses shows that a relatively simple modified Coulomb type friction model with a steep gradient through the origin delivers sufficiently accurate results. The computations reveal the existence of 'locked' zones with extremely low 'creeping' motions.

Thus, the mechanisms of power transmission in belt-type CVT are better understood and designers have a validated tool to optimize shaft and disc geometry concerning maximum efficiency.
\end{abstract}

Keywords: continuously variable transmissions, efficiency, coefficient of friction, sliding motion

\section{INTRODUCTION}

Belt-type continuously variable transmission (CVT) units are gear units with transmission by traction forces. They comprise two parallel shafts, each with one fixed disc and one moveable disc. Axial displacement of the moveable disc forces the chain to contact the discs on a different running perimeter. The ratio of transmission changes and the input speed and torque are transformed (Fig. 1). The subassembly that comprises both shafts and the chain is referred to as the variator.

In most applications, the normal force required for power transmission is generated using a hydraulic clamping system. It also provides oil to lubricate the

\footnotetext{
${ }^{*}$ Corresponding author: Department of Machine Elements, Institute for Machine Elements, Engineering Design and Tribology, Welfengarten 1A, Hannover 30167, Germany. email: poll@imkt.uni-hannover.de
}

traction contact between the discs and the chain. The forces applied to the arc of contact and the limited disc stiffness impose elastic deformations on the discs and shafts and thereby deflections, which vary along the perimeter (Fig. 2). The necessary clearance between the moveable disc and the shaft causes the moveable disc to tilt. Furthermore, the normal forces compress the chain. All these effects induce sliding movements between the chain and the discs, or what is known as a spiral path, which is largely responsible for the loss of power and wear in the variator (Fig. 3) $[\mathbf{1}, 2]$. Therefore, the performance of the variator can be improved by optimizing its geometry with respect to deflections.

\section{BASES FOR A NUMERICAL MODEL}

To design a highly efficient yet compact variator, it is important to understand the key influencing 


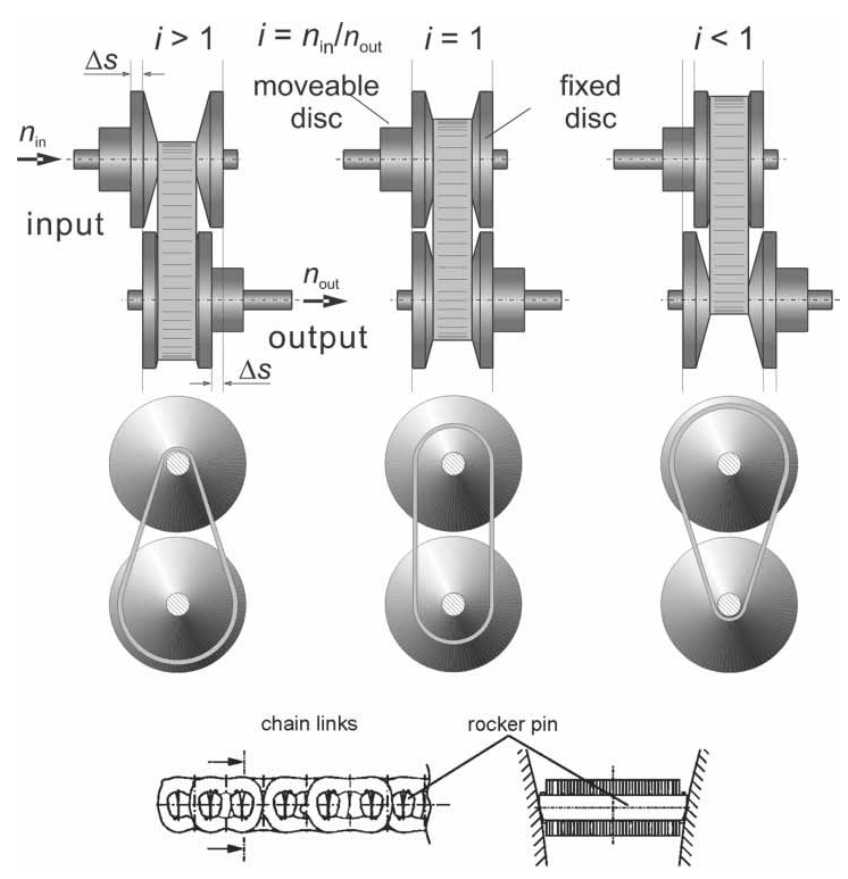

Fig. 1 Functional principle of variable belt transmission [2] and rocker pin chain [7]

variables in the arc of contact, such as the sliding velocity and sliding direction, the longitudinal tensile force in the chain, and the force components transmitted between discs and pins. The loss of power and the resultant efficiency can be calculated from these quantities. A simulation programme has been developed for this purpose. It can be used at a very early stage of the development process, i.e. as soon as the first geometric variant of the variator has been devised. The results are used for determining the ideal geometry of the variator and also help to determine the best way to design the other components of the transmission unit, e.g. hydraulics. The simulation is based on the following assumptions $[2,3]$.

1. The longitudinal strain and the transverse compression are independent of each other. This strictly applies to the rocker pin element chain because the functions of the rocker pin elements (axial forces) and links (peripheral forces) are separated, which means that the compression in the rocker pin elements does not have any influence on the longitudinal strain in the links. Likewise,

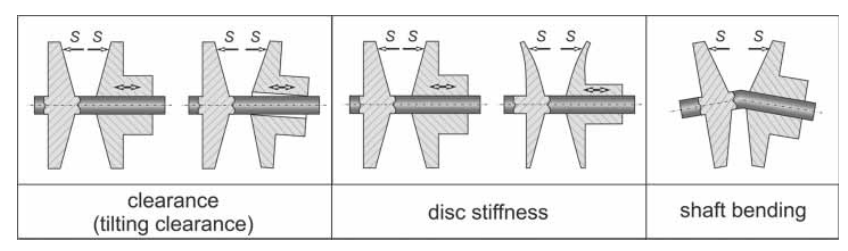

Fig. 2 Causes of sliding movements and losses

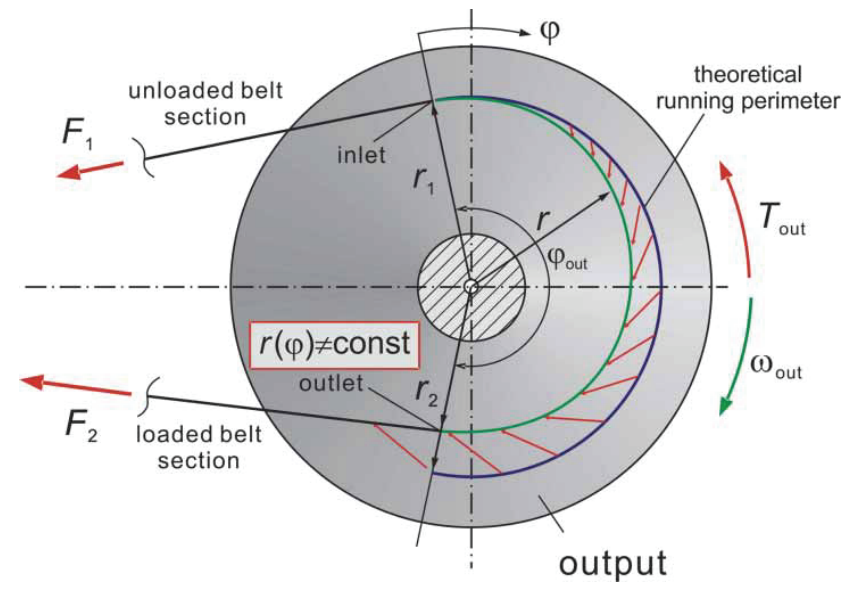

Fig. 3 Spiral path [2, 9]

the longitudinal strain in the links has no effect on the normal forces in the pin elements.

2. The chain is a continual element with even mass distribution and longitudinal and transverse stiffness.

3. The wedge-shaped gap between the discs is concerned as symmetrical. Both contacts (with the moveable disc and the fixed disc) have the same contact conditions. The deformations on the driving disc and the moveable disc are of a similar magnitude. Although the two discs may deflect and tilt differently, the error when assuming equal contact angles on both sides can be concerned as negligible.

4. Coulomb's law of friction applies except for very low-sliding speed. The friction coefficient has a constant mean value that is derived from the measured values. A continuous analytic function is selected for areas in which sliding speeds are very small (discussed subsequently).

5. Internal losses in the chains are not taken into account. The rocker pin element chains have a very low level of internal losses. Specific functional approaches have to be added to determine internal losses in push belts [4].

6. The same approaches are used for the driving and the driven set of discs.

Figure 4 shows the sliding velocity $\left(\bar{V}_{\mathrm{sl}}=\bar{V}_{\text {disc }}-\right.$ $\left.\bar{V}_{\text {chain }}\right)$ and force vectors in the contact between the tapered disc and the chain in a tapered disc increment with a size of $d \varphi$ and a taper angle of $\beta$. The normal force $\mathrm{d} N$ between the chain and the disc acts perpendicular to the surface. The varying longitudinal stretching of the chain causes sliding movements in the direction of the periphery when tensile force is built up or reduced.

In addition there is radial sliding and as a consequence, the running radius changes. The sliding angle $\gamma$, which in the mathematically positive sense 


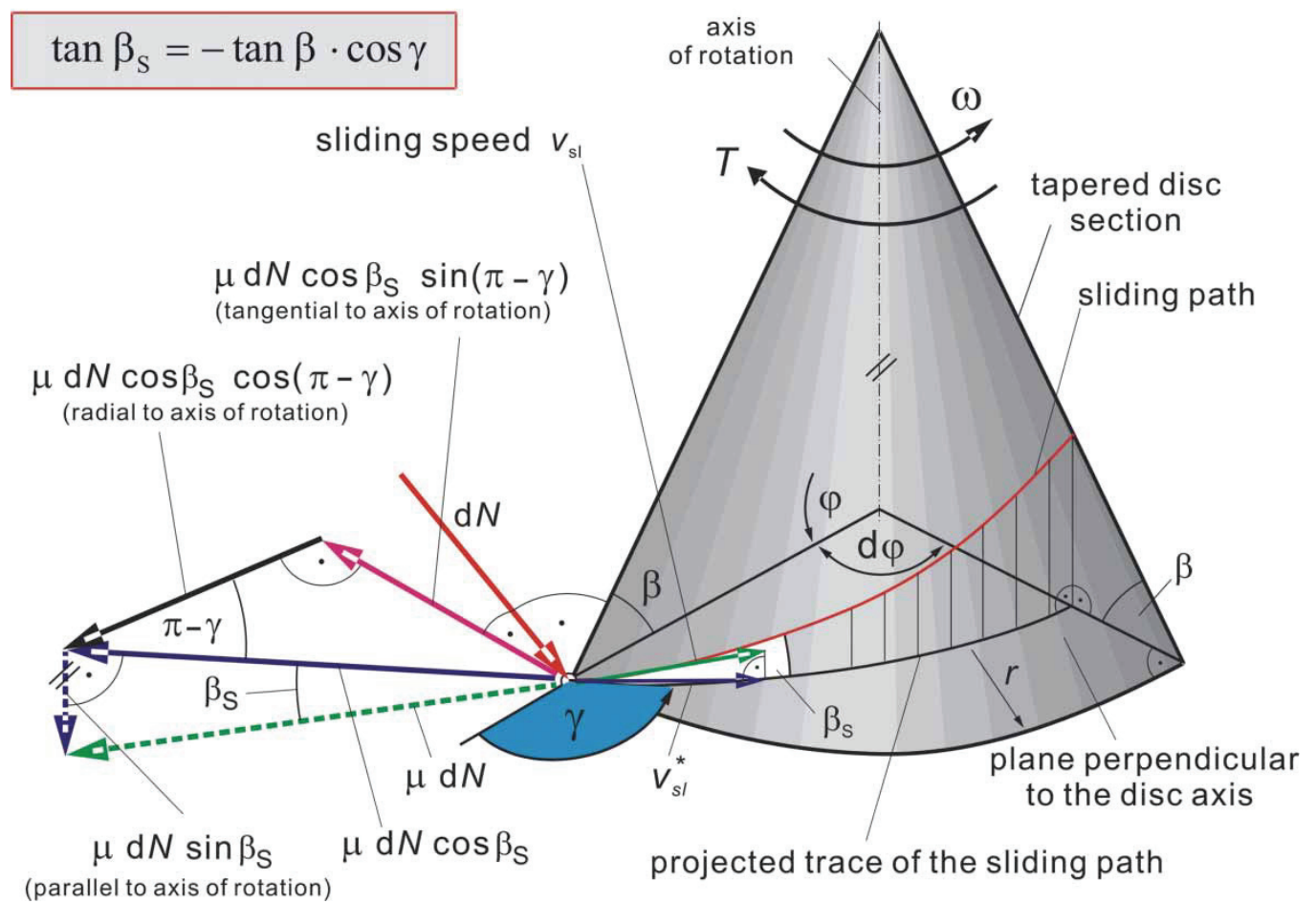

Fig. 4 Sliding and force vectors in the contact [3]

is measured against the outward pointing radial beam, denotes the direction of the sliding movement. The effective taper angle $\beta_{\mathrm{S}}$ indicates the spatial direction of the sliding movement. The force of friction on the chain is directed opposite to the sliding velocity vector $\boldsymbol{v}_{\mathrm{sl}}$ and is the product of the normal force and the local friction value $\mu \mathrm{d} N$. This formally corresponds to Coulomb's law of friction. However, in reality, there may be different friction levels in the contact between the chain and the discs depending on the position of the arc of contact and the peripheral velocity.

The adhering layer of lubricant is first entrained in the chain's inlet zone into the wedge-shaped gap and subsequently gradually squeezed out of the contacts. This then results in mixed lubrication and finally boundary lubrication. Calculating the thickness of the lubricating films, the temperature-related viscosity and the resultant fluid friction and boundary friction ratio are complicated tasks. Therefore, for an initial approximation, a uniform and location-independent law of friction is adopted with which the friction value for low-sliding velocities tends towards zero (1)

$$
\mu=\mu_{\mathrm{sl}} \cdot\left(1-\mathrm{e}^{-v_{\mathrm{sl}} / \nu_{0}}\right)
$$

Figure 5 shows the applied function for low-sliding velocities $\left(\boldsymbol{v}_{\mathrm{sl}}<1 \mathrm{~mm} / \mathrm{s}\right)$ with a sliding coefficient of

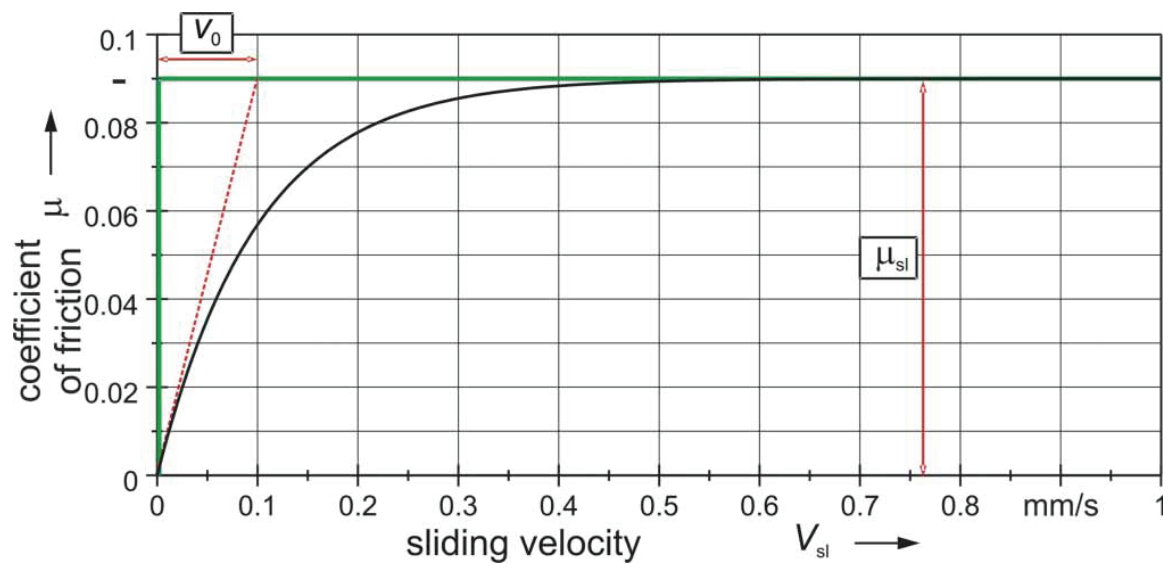

Fig. 5 Friction law at low-sliding velocities 
friction $\mu_{\mathrm{sl}}=0.09$ and a speed constant $\nu_{0}=0.1 \mathrm{~mm} / \mathrm{s}$ [5]. Thus, the static coefficient of friction is zero; instead of genuine adhesion, creeping takes place at very low-sliding velocities. This type of behaviour seems physically very plausible, although it has yet to be confirmed in experiments [6].

This approach has the numerical advantage that adhesion conditions do not have to be checked and there is always a small sliding velocity with which the sliding angle can be determined. The force of friction can be separated into a radial component $\left(\mu \cdot \mathrm{d} N \cdot \cos \beta_{\mathrm{S}} \cos (\pi-\gamma)\right)$ and a tangential component $\left(\mu \mathrm{d} N \cdot \cos \beta_{\mathrm{S}} \cdot \sin (\pi-\gamma)\right)$, which causes the tensile force to change. Figure 6 shows the radial and tangential components of the force of friction. The equation for $\mathrm{d} N$ results after transformation, neglecting the change in the radius of curvature. It is important to take into account that the axial components count twice because of the two-pulley chain contacts.

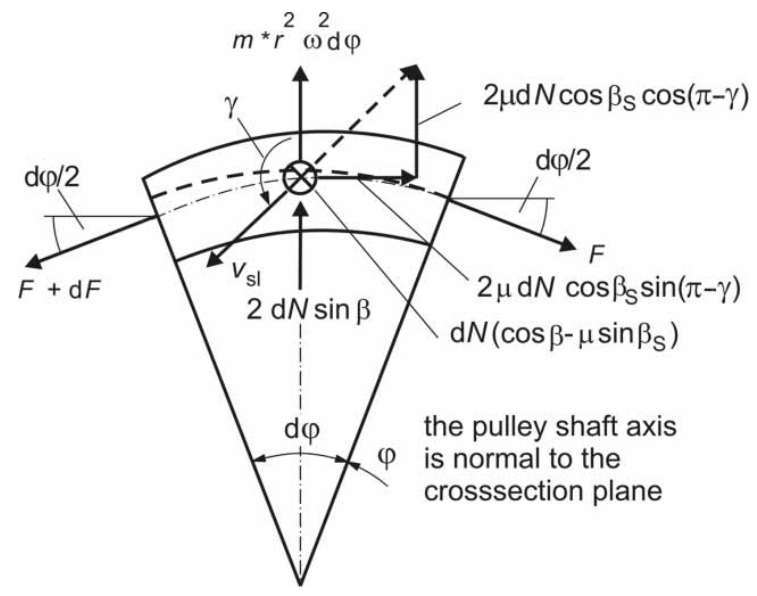

Fig. 6 Forces acting on the arc element in the axial section
In this way, a system of first-order differential equations can be established for the calculation (Fig. 7). The motion relationship in the arc of contact can be described with these equations. As all coupled variables are known at the outset or can be described by analytical functions, the calculation can be considered as an initial value problem and solved according to the Runge-Kutta procedure of fourth order for coupled systems of differential equations $[2,3]$.

The local taper angle $\beta$ and widening $w$ of the wedge-shaped gap between the discs are determined by finite-element calculations. Two-dimensional finite-element models for the half-section of the variator suffice for these calculations, because the selected element type and rotationally symmetrical components allow to apply stresses which are not axially symmetrical. Following the finite-element calculation, the axial deformation and the actual tapered angle are determined at discrete points on the running radius and approximated with a polynomial function, delivering the variables as a function of the arc angle.

\section{SOLUTION BY ITERATIVE COMPUTATION}

The computation of an operating point is an iterative process, and the calculation is made first for the output set of discs. For this purpose, starting values are specified for the starting tensile force $F_{0}$ and the starting sliding angle $\gamma_{0}$, and an initial deformation is specified. The nominal axial force $S_{2, \operatorname{targ}}$ and the nominal drive torque $T_{2, \text { targ }}$ serve as target values. If no solution is achieved after a first calculation, the starting values $\left(F_{0}, \gamma_{0}\right)$ are varied such that better agreement with the target values is obtained (Fig. 8).

$$
\begin{array}{ll}
\text { tensile force: } & F^{\prime}=\frac{\mathrm{d} F}{\mathrm{~d} \varphi}=\left(F-m^{*} \cdot r^{2} \cdot \omega^{2}\right) \cdot \frac{\mu \cdot \cos \beta_{\mathrm{S}} \cdot \sin \gamma}{\sin \beta-\mu \cdot \cos \beta_{\mathrm{S}} \cdot \cos \gamma} \\
\text { sliding angle: } & \gamma^{\prime}=\frac{r^{\prime}-\left(\mathrm{K}_{1} \cdot(\Theta+\Phi)+\mathrm{K}_{4} \cdot \Psi\right) \cdot \beta^{\prime}-\mathrm{K}_{3} \cdot(\Theta+\Phi) \cdot w^{\prime}-\mathrm{K}_{6} \cdot \Psi}{\mathrm{K}_{2} \cdot(\Theta+\Phi)+\mathrm{K}_{5} \cdot \Psi} \\
\text { running perimeter: } & r^{\prime}=\left(\frac{F+E A}{F_{0}+E A} \cdot\left(r_{0}+r_{0}^{\prime} \cdot \tan \gamma_{0}\right)-r\right) \cdot \frac{1}{\tan \gamma} \\
\text { torque: } & T^{\prime}=-F^{\prime} \cdot r \\
\text { clamping force: } & S^{\prime}=\frac{\mathrm{d} S}{\mathrm{~d} \varphi}=\left(F-m^{*} \cdot \omega^{2} \cdot r^{2}\right) \cdot \frac{\cos \beta-\mu \cdot \sin \beta_{\mathrm{S}}}{2 \cdot\left(\sin \beta-\mu \cdot \cos \beta_{\mathrm{S}} \cdot \cos \gamma\right)} \\
\text { power losses: } & P_{\mathrm{V}, \mathrm{Var}}^{\prime}=\frac{\mathrm{d} P_{\mathrm{V}, \mathrm{Var}}}{\mathrm{d} \varphi}=2 \cdot\left|v_{\mathrm{g}}\right| \cdot \mu \cdot \frac{\left(F-m^{*} \cdot \omega^{2} \cdot r^{2}\right)}{2 \cdot\left(\sin \beta-\mu \cdot \cos \beta_{\mathrm{S}} \cdot \cos \gamma\right)} \\
\text { numerical solution: } & \text { Runge Kutta of } 4^{\text {th }} \text { order for coupled sets of differential } \\
\mathbf{w}(\varphi), \boldsymbol{\beta}(\varphi): & \quad \begin{array}{l}
\text { equations (see appendix) } \\
\text { FE-computation }
\end{array}
\end{array}
$$

Fig. 7 Differential equations system [10] 

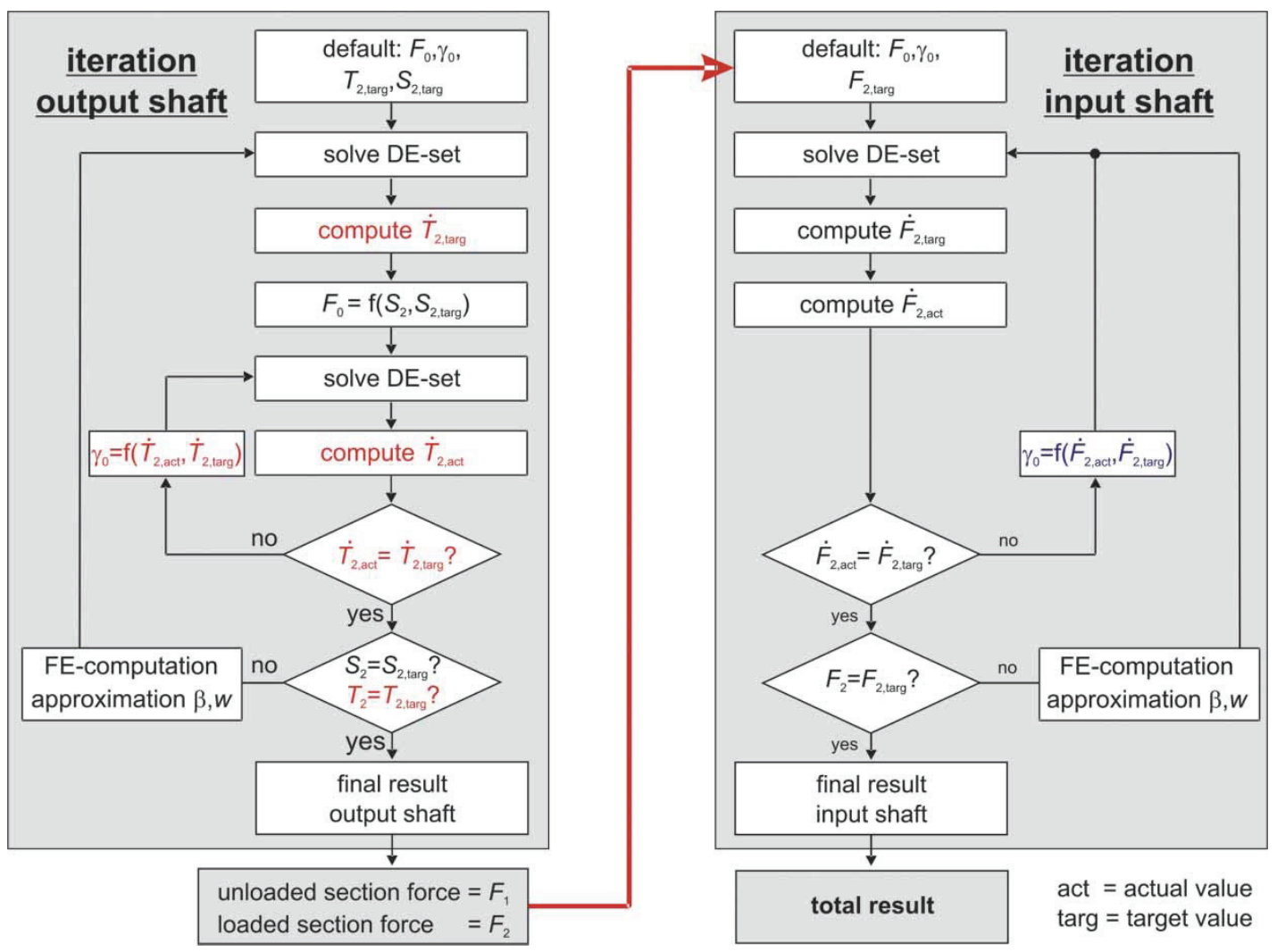

Fig. 8 Computation scheme for driving and driven discs $[2,10]$

Only the torque is known at the input set of discs. In the real gear unit, the clamping force is varied such that the nominal transmission ratio is obtained. As no change of ratio operations can be simulated with the calculation procedure and the clamping force at the drive is not known a priori, a different boundary condition must be found. Both disc sets are in mutual contact only via the chain. After the calculation for the driven set of discs, the belt tension forces are known and can thus be used as boundary condition and target values for the calculation on the driving side. The tensile force at the output of the driven set of discs is the tensile force at the input of the driving set of discs. Consequently, the starting tensile force at the driving set of discs is not available as variation parameter for solving the set of differential equations. However, only one boundary condition, namely, the final tensile force $F_{2}$, still has to be fulfilled. The starting sliding angle remains as variation parameter. For the driving discs, as already for the driven discs with the torque, the tensile force is not used for the variation, but instead the tensile force changes as reference variable and controlled variable.

The possibilities of the procedure will now be explained with the help of an example. The tensile force $F$, the local axial force $S^{\prime}$, and the local power transmission losses $P_{\mathrm{V}, \mathrm{Var}}^{\prime}$ are shown in Fig. 9 over the arc of contact at the two disc sets. The arrows indicate the direction of the sliding movements (sliding angle $\gamma$ ), and the arrow length corresponds to the magnitude of the sliding velocity.

The transmitted torque is increased in steps starting from zero. Without torque, the tensile forces are identical in both belt sections. For $i=1$, the clamping forces at the driving and driven discs are also equal; consequently, identical conditions must exist at both disc sets. The sliding movement is directed inwards and backwards at the input. Over the arc of contact, the vector changes direction through $180^{\circ}$ (purely inward sliding at the orthogonal point). The sliding velocity becomes smaller.

A well-recognizable region of very small, almost constant, sliding velocity appears. In this 'creep zone', genuine adhesion could appear if the static coefficient of friction would not be zero at the origin. Increased outward drifting appears as the end of the arc of contact is approached. A stronger increase in the axial force also takes place here, causing high local power transmission losses. The losses are almost zero in the adhesion region. As the torque is increased, the axial force peak at the driven discs flattens and moves in the direction towards the input. The region of small sliding velocity becomes smaller and is also displaced in the direction towards the input. The sliding angle is increasingly directed backwards. 

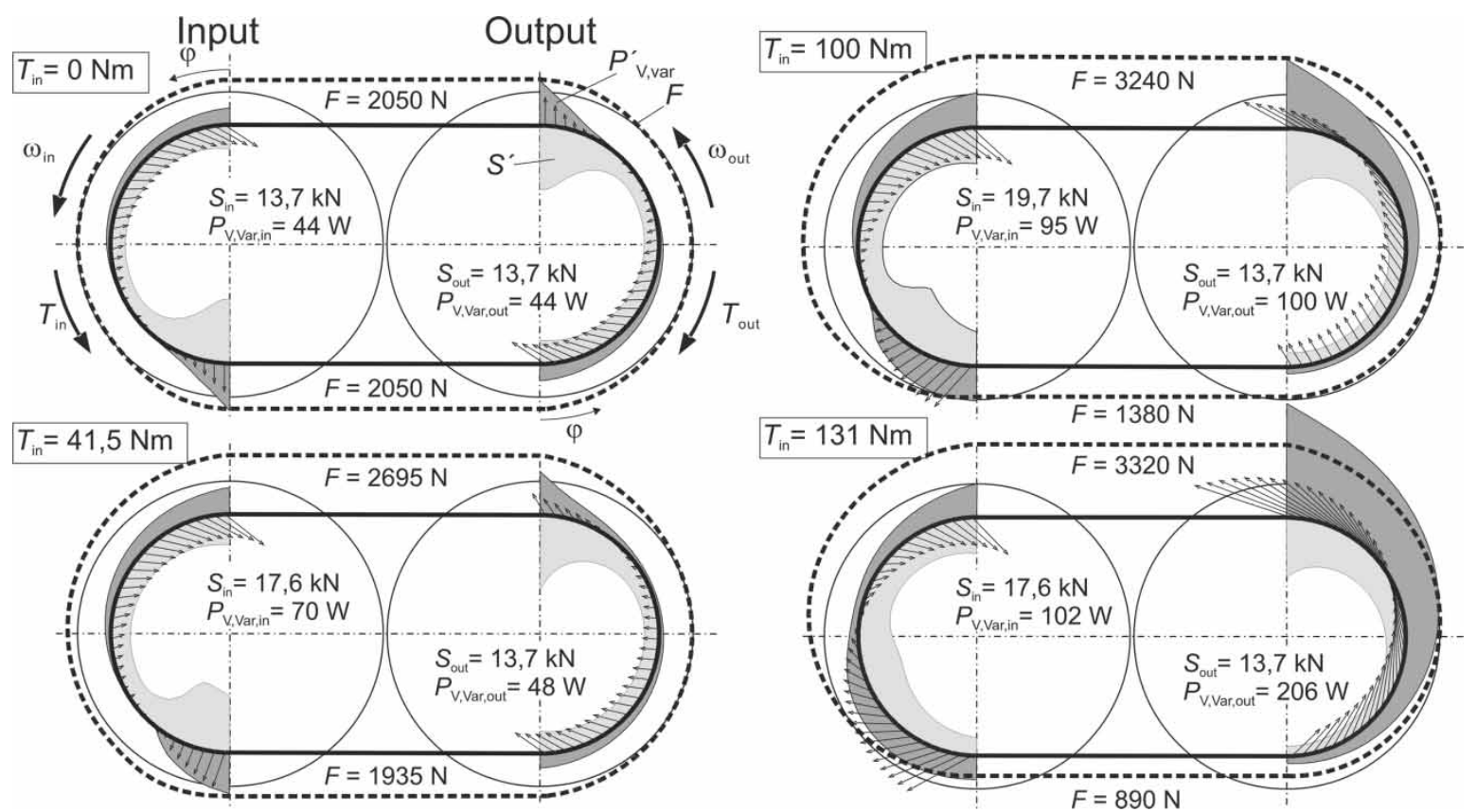

Fig. 9 Calculated forces, power losses, and sliding velocities over the arc of contact on increasing the drive torque $[\mathbf{2}, \mathbf{1 0}]$

The fundamental form of the axial force distribution changes hardly at the driven discs, but its maximum drifts in the direction towards the outlet. The sliding angle turns into a forward at the inlet. In the last section of the figure $(131 \mathrm{Nm})$, the sliding angle is directed forward at the driven disc and backward at the driving disc along both contact arcs. The large increase in the power transmission loss indicates that the chain is close to the onset of gross slipping.

\section{VERIFICATION}

Computations were compared with experimental results of the authors for verification. Figure 10 shows the power transmission efficiency and power loss from one measurement and two calculations. The power transmission loss curves run approximately parallel with increasing torque up to a nominal torque of $120 \mathrm{Nm}$, whereby the

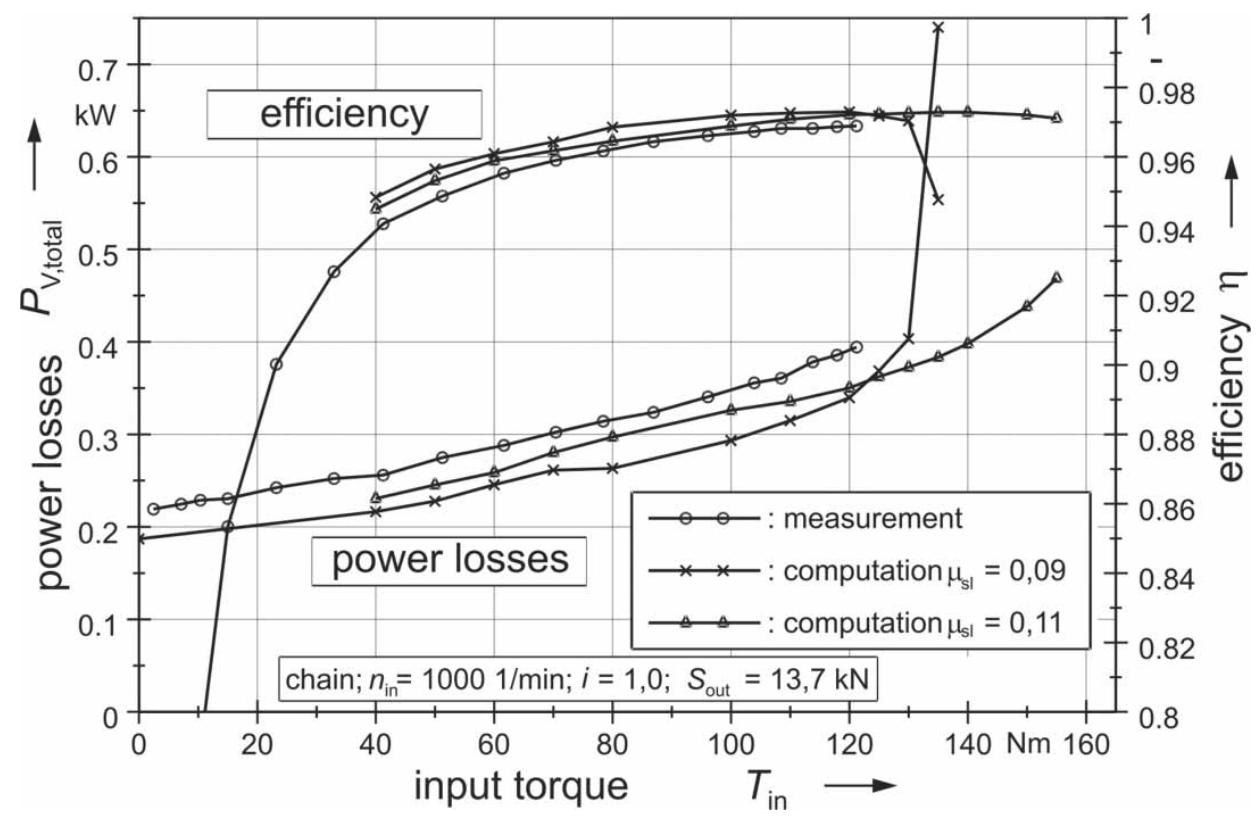

Fig. 10 Comparison of measurements and calculations for different friction coefficients 
calculated values lie slightly below the measured values.

The curve with the friction coefficient of $\mu_{\mathrm{sl}}=0.11$ approaches the measured curve more closely. As from $\sim 120 \mathrm{Nm}$, the calculated power transmission losses for a friction coefficient of $\mu_{\mathrm{sl}}=0.09$ increase strongly and the power transmission efficiency falls. This indicates that the chain is close to the onset of gross slipping. In contrast thereto, the power transmission efficiency maximum of the calculation variant with a friction coefficient of $\mu_{\mathrm{sl}}=0.11$ is reached only at $\sim 135 \mathrm{Nm}$. Although the power dissipated with a friction coefficient of $\mu_{\mathrm{sl}}=0.11$ evidently matches the real system more closely, the

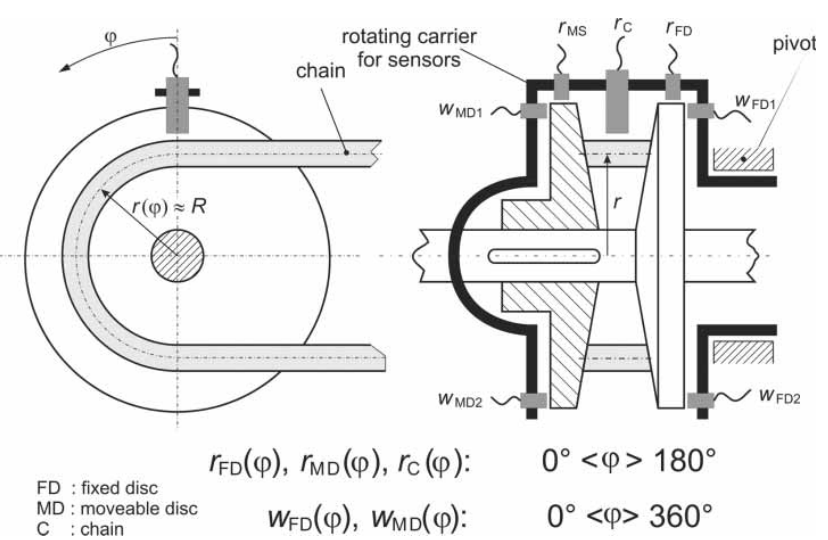

Fig. 11 Measuring system for determining the shaft and disc deformations influence of the assumed sliding friction coefficient is surprisingly small in the range of practical interest. Only the threshold torque for the onset of slipping responds sensitively as expected. The approximation with a simple friction law already represents reality very closely.

An explanation for this is that the friction has two opposite effects that mutually cancel to a large extent in the system considered here. With small friction coefficients, the local friction forces become smaller, but the sliding movements increase at the same time, so that the frictional work hardly changes.

The reasons of the variator deformations described earlier were investigated by measurements on various shaft and disc geometries, so that measurement results are available for various shaft stiffness values and tilt play variants. For this purpose, a measuring system was integrated in a test gear unit (Fig. 11).

The disc deformations are measured with six distance sensors (three each on the fixed disc and on the moveable disc; right part of the figure), which are mounted on a measuring device. The measuring device is pivot mounted on the bearing pots and, as there are two sensor sets shifted by $180^{\circ}$ with respect to each other, the deformation can be measured over the entire circumference. A sensor on the left part of the figure determines the position of the chain in the region of the arc of contact.

Figure 12 shows an example of the comparison between the measurement (black line) and the calculation (grey line) for the driving and driven discs with transmission ratio $i=1$ and a driving torque of $120 \mathrm{Nm}$. The chain runs at the top at $90^{\circ}$ into the disc

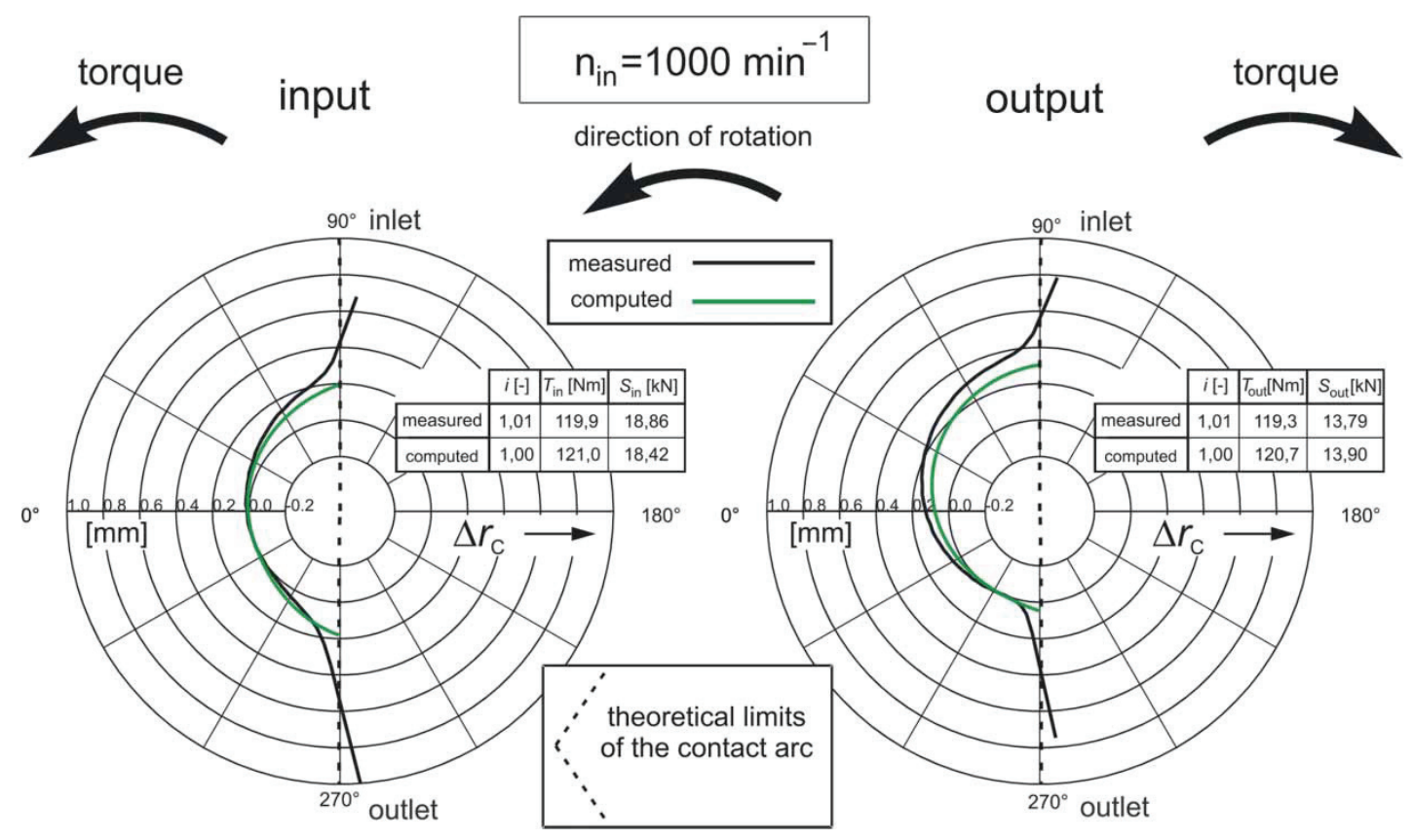

Fig. 12 Comparison of measured and calculated deformations [2] 


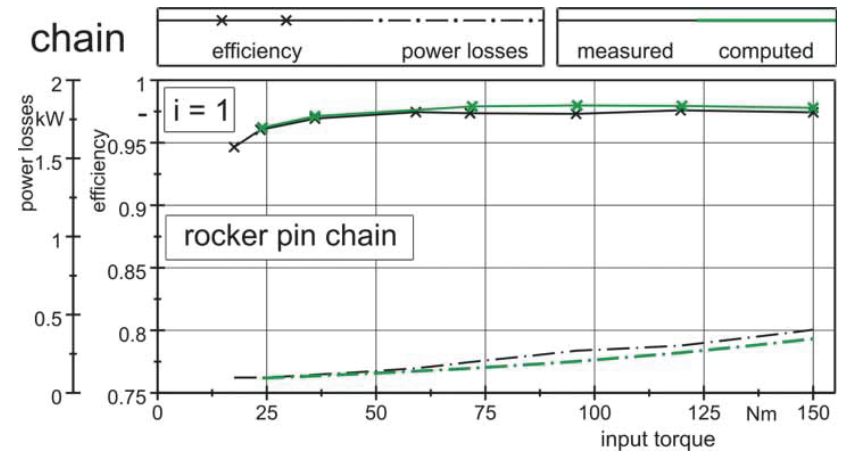

Fig. 13 Comparison between loss and efficiency measurements and calculations [2]

and at the bottom at $270^{\circ}$ out of the disc set again. The different measured characteristics at the driving and driven discs are reproduced well by the calculation. This makes it reasonable to conclude that the axial forces and their distribution over the arc of contact come very close to the real conditions, albeit the calculated curves lie slightly below the measured values.

Power transmission efficiency measurements were carried out for the same variator execution in addition to the deformation measurements. The measured and calculated results for the power transmission efficiency and for the power loss are plotted in Fig. 13 against the drive torque, with a transmission ratio of $i=1$ and a rotation speed of $n=1000 \mathrm{r} / \mathrm{min}$. The respective curves match closely. Again, a friction coefficient of $\mu_{\mathrm{sl}}=0.11$ was used in the computations, also resulting in a good match for the axial clamping force required to maintain the transmission ratio. The model has been validated with measurements at three different transmission ratios, several rotational speeds, four different geometries of the discs, and two different tilting clearances of the moveable discs. The accuracy margin of the measurements is 0.11 per cent.

\section{SUMMARY}

Owing to deformations, sliding movements take place in variators of chain type CVT. They are largely responsible for the power transmission losses. Measurements and calculations show that the operational behaviour of continuously variable belt transmissions can be reproduced accurately even when a very simplified friction law is taken as basis. This requires an iterative computation method based on a system of differential equations and finite-element calculations. The distribution of the axial forces and the deformations of all variator components can be reproduced realistically therewith, making it a useful tool for variator optimization already in the early design stage.

\section{REFERENCES}

1 Dittrich, 0. Anwendung der Theorie des keilförmigen Umschlingungstriebes auf stufenlose Getriebe. Bad Homburg, 1992.

2 Sue, A. Betriebsverhalten stufenloser Umschlingungsgetriebe unter Einfluss von Kippspiel und Verformungen. Doctoral Thesis, Hannover University, IMKT 2003.

3 Sattler, H. Stationäres Betriebsverhalten stufenlos verstellbarer Metallumschlingungsgetriebe. Doctoral Thesis, Hannover University, IMKT 1999.

4 Sladek, W. Möglichkeiten zur Wirkungsgradoptimierung von Stufenlosgetrieben. Doctoral Thesis, Hannover University, IMKT 2001.

5 Srnik, J. Dynamik von CVT-Keilkettengetrieben. Doctoral Thesis, TU Munich, 1998, VDI-Fortschritt-Bericht No. 372 Reihe 12, (VDI-Publishing, Düsseldorf).

6 Wang, G. Untersuchungen an stufenlos einstellbaren Zug- und Schubgliedergetrieben. Doctoral Thesis, TU Clausthal, 1991.

7 Faust, H. and Linnenbrügger, A. CVT-Entwicklung bei LuK. 6th LuK Kolloquium, 1998, pp. 159-181 (LuK, Bühl).

8 Sattler, H. Efficiency of metal chain on V-Belt CVT. International Congress on Continuously variable power transmission, Eindhoven University of Technology, 1999, pp. 99-104.

9 Sue, A. Dittrich, O., and Poll, G. CVT-Wirkungsgradberechnung aus der Verformung beim Umschlingungstrieb. CVT 2002 Congress, VDI-reports Vol. 1709, 2002, pp. 69-88 (VDI-Publishing, Düsseldorf).

10 Sue, A. and Poll G. Analytisch-iteratives Berechnungsverfahren für stufenlose Umschlingungsgetriebe. VDIreports Vol. 1827, 2004, pp. 691-712 (VDI-publishing, Düsseldorf).

\section{APPENDIX 1}

\section{Notation}

$F_{0}$

$F_{1} \quad$ force in the unloaded belt section

$F_{2} \quad$ force in the loaded belt section

$i \quad$ ratio

$P_{\mathrm{V}, \mathrm{Var}}^{\prime} \quad$ local power losses in the variator

$q \quad$ stiffness

$R \quad$ running perimeter

$S \quad$ clamping force

$S^{\prime} \quad$ local clamping force

$T \quad$ torque

$T \quad$ local torque

$\boldsymbol{v}_{\mathrm{sl}} \quad$ sliding velocity

$\beta \quad$ tapered disc angle

$\beta_{\mathrm{S}} \quad$ effective tapered disc angle

$\gamma \quad$ sliding angle

$\gamma_{0} \quad$ starting sliding angle

$\mu_{\mathrm{sl}} \quad$ coefficient of friction

$\omega$ revolution speed 


\section{APPENDIX 2}

$\Theta=\frac{q \cdot E}{(A B-C D)^{2} \cdot H J}$

$\Phi=\frac{q^{2} \cdot A E^{2}}{(A B-C D)^{2} \cdot H J \cdot \sqrt{(q \cdot A E)^{2}-F \cdot b \cdot \mathrm{C} \cdot(A B-C D)}}$

$\Psi=\frac{F \cdot b}{2 \cdot(A B-C D) \cdot H J \cdot \sqrt{(q \cdot A E)^{2}-F \cdot b \cdot C \cdot(A B-C D)}}$

$K_{1}=L \cdot(A B-C D)-A \cdot(4 q \cdot A H+B L)+A D N$

$K_{2}=M \cdot(A B-C D)-A B M+A D O$

$K_{3}=-A \cdot(A B-C D) \cdot \frac{H J}{E}$

$K_{4}=-N \cdot(A B-C D)+C \cdot(4 q \cdot A H+B L)$

$-C D N$

$$
\begin{aligned}
& K_{5}=-O \cdot(A B-C D)+B C M-C D O \\
& K_{6}=\left(\frac{r \cdot r \cdot D}{(F \cdot b)-1}\right) \cdot(A B-C D) \cdot C G H J / A \\
& A=\sin \beta-\mu \cdot \cos \beta_{\mathrm{S}} \cdot \cos \gamma \\
& B=4 \cdot q \cdot \tan \beta \\
& C=\cos \beta-\mu \cdot \sin \beta_{\mathrm{S}} \\
& D=m^{*} \cdot \omega^{2} \cdot b \\
& E=2 \cdot R \cdot \tan \beta_{0}-w \\
& G=\mu \cdot \cos \beta_{S} \cdot \sin \gamma \\
& H=1+\tan ^{2} \beta \cdot \cos ^{2} \gamma \\
& J=\cos ^{2} \beta \\
& L=-\mu \cdot \sin \beta_{S} \cdot \cos ^{2} \gamma+\cos \beta \cdot H \cdot J \\
& M=\mu \cdot \sin \beta \cdot \cos \beta \cdot \sin \beta_{S} \cdot \sin \gamma \cdot \cos \gamma+G \cdot H \cdot J \\
& N=\mu \cdot \cos \beta_{S} \cdot \cos \gamma-\sin \beta \cdot \cos ^{2} \beta \cdot H \\
& O=-\sin \beta \cdot \cos \beta \cdot G
\end{aligned}
$$

